\title{
Near Optimal Linear-Service Oriented Resource Allocation Strategy for LTE Networks
}

\author{
Ahmad Shokair* ${ }^{*}$, Youssef Nasser ${ }^{\dagger}$, Oussama Bazzi ${ }^{\ddagger}$, Jean-Francois Helard* and Matthieu Crussiere* \\ * IETR / INSA de Rennes, 20 Ave. des Buttes de Coësmes, Rennes, France \\ \{ashokair, mcrussie, jfhelard\} @insa-rennes.fr \\ $\dagger$ American University of Beirut, Beirut, Lebanon 1107 2020, yn10@aub.edu.lb \\ ¥ Lebanese University, Faculty of Sciences, Rafic Hariri University Campus, Hadath, Lebanon, obazzi@ul.edu.lb
}

\begin{abstract}
Extensive research efforts are being attracted towards resource allocation in LTE networks. However, from the resource allocation perspectives, linear services such as Digital TV programs have been scarcely studied. In fact, for end-users requesting linear services, the maximization of the the number of users served is much important goal than maximizing the overall capacity of the network. In this paper, linear services oriented resource allocation strategy is proposed. The goal of the proposed algorithm is to maximize the success rate of the users, depending on a minimum required quality of service $(\mathrm{QoS})$, measured in terms of user capacity constraint. The proposed method was tested and compared to the optimal branch and Cut solution and has shown good robustness against network and user parameters.
\end{abstract}

\section{INTRODUCTION}

Lately, versatile users associated with broadband systems have been increasingly watching linear services on their cell phones and tablets. Linear services could be characterized as the video services where the end-client watches a program controlled totally by the transmitter. Thusly, broadband systems (such as LTE) offering these services need to deal with a colossal measure of data activity [1]. Additionally, these systems need to deal with the signal to interference and noise ratio (SINR) due to the inter-cell interference and also the tremendous measure of data for such services.

Measures like frequency reuse methods were taken in an attempt to reduce interference, and consequently, increase the average capacity of a user. However, frequency reuse will have a narrow effect since the increase in capacity has logarithmic relation with SINR that frequency reuse offers to enhance. On the other hand, managing the bandwidth for each user affects linearly the users capacity, so it is very important to efficiently manage the overall resource allocation so that certain requirements like rate, minimum user capacity, and fairness, etc, are satisfied.

Unicast transmission of non-linear services has taken extensive research. The reader may refer to the works in [2-5]. However, to the best of the authors' knowledge, none of these strategies has dealt with linear services which require a maximization of the number of users served. Indeed, in contrary to regular services where increasing a user's capacity would enhance the Quality of Service (QoS), the allocation of larger number of resource blocks to the end-users will not be reflected in QoS . Indeed, a user receiving linear service needs a minimum capacity level to properly receive the content, making it inefficient to allocate resources beyond this level. As a result, system capacity enhancement would be a secondary objective when compared to enhancing the percentage of users receiving a linear service. Another important factor resides in the utilization rate. Indeed, in non-linear services, existing methods exploit all the available RBs, while in linear services case, other services might need resources to be allocated. This affects different resource allocation outcomes, such as power reduction, new arrivals, congestion, etc. Hence, it is very important to propose fair allocation approaches when dealing with linear services.

In literature, Round Robin (RR) algorithm, among others, is exhibited as the fairness approach. In RR, Resource Blocks (RB) are iteratively distributed to the distinctive users until all are allocated, or the user's constraints are met [6]. This guarantees that all users are relegated with RBs. However, RR doesn't consider the channel conditions for every client, so it fails to accomplish high rates coverage. Conversely, the Maximum Throughput (MT) approach allocates the first RB to the user with the best channel conditions, then moves to the second $\mathrm{RB}$, and reruns the distribution until all RBs are designated or the user's limitations are fulfilled. This strategy guarantees greater achievable rate at the detriment of fairness, since a user with best channel conditions could acquire the majority of the RBs, leaving different users with limited or even no RBs. Nonetheless, this technique could be utilized as a benchmark for system capacity. As a compromise between the two above methods, Proportional Fair (PF) [7] allocates resources according to a formula that considers not only channel conditions of a user, but also the number of resource blocks already allocated to that user.

In this work, we firstly derive and model the resource allocation problem of a linear service as a Mixed Integer Programming (MIP) problem. Then, we present a new Linear Service Oriented Resource Allocation Strategy (LSORAS), that aims to maximize the percentage of users receiving the linear service while maintaining a minimum required capacity for each user, with decent fairness level. This guarantees that each user will be allocated the required number of RBs hence, granting resources to other services, or idling unused RBs. The proposed algorithm is compared to RR, PF, MT methods, and more importantly to the optimal allocation provided by the 
branch and cut $(\mathrm{BaC})$ optimization technique usually adopted for MIP problems. Average success rate (SR) has been mainly used to assess the two algorithms performance. Moreover, the effect of user density and required capacity on the SR was analyzed.

It should be noted that linear services should be offered by a broadcasting/multicast system or a broadcasting mode such as the MBMS mode in LTE networks. However, as the network operators are using unicast mode for most of their services, it is judicious to model and analyze this mode for linear services. Hence, the importance of the current work is in the system specifications for network operators that need to offer linear services.

The rest of this paper is organized as follows: In section II, the used model, the assumptions, and the performance metrics are introduced. Section III presents the problem and the new proposed algorithm. In section IV the simulation results are shown and section V concludes this work.

\section{MODEL DESCRIPTION}

We consider in this work an LTE network using Orthogonal Frequency Division Multiplexing (OFDM) technology for the transmission of linear services. we assume that $M$ users are uniformly distributed in the service area, by the means of $N$ BS sites forming the Broadband network.

\section{A. Requirements and Assumptions}

In this work, linear service is assumed to be always available to any user at any time. A minimum capacity $C_{r e q}$ is required for a user to properly receive the service, i.e. $C^{\text {user }} \geq C_{\text {req. }}$. In the broadband network, unicast is used to separately deliver the linear service to each connected user. It is also assumed that the user is connected to the nearest BS, and the transmission power is considered to be identical in every cell. Another assumption is that the BS has complete information about the channel with each user within the cell borders.

The BSs are considered to be uniformly distributed over the service area, according to a Poisson Point Process (PPP) with density $\lambda_{B S}$, where Voronoi tessellation is employed to draw the cell borders. The users' density is also assumed to follow PPP with density $\lambda_{u}$. A diagram representing the service area, including base stations and users, is shown in Fig.1. The edge of the service area is represented by the red circle, connections between a BS with its served and un-served users is represented with black and cyan lines respectively.

\section{B. Broadband model}

A number of RBs is allocated for each connected user by its serving BS. The transmission bandwidth controls the total number of RBs available at a BS transmitter, which is denoted by $R B_{\max }$.

Hence, the SINR for user $m$ at RB $i$ is given by:

$$
\gamma_{m, i}=\frac{P_{t} h_{m, i}\left(\frac{4 \pi f r_{m}}{c}\right)^{-\alpha}}{\sigma^{2}+I_{m, i}}
$$

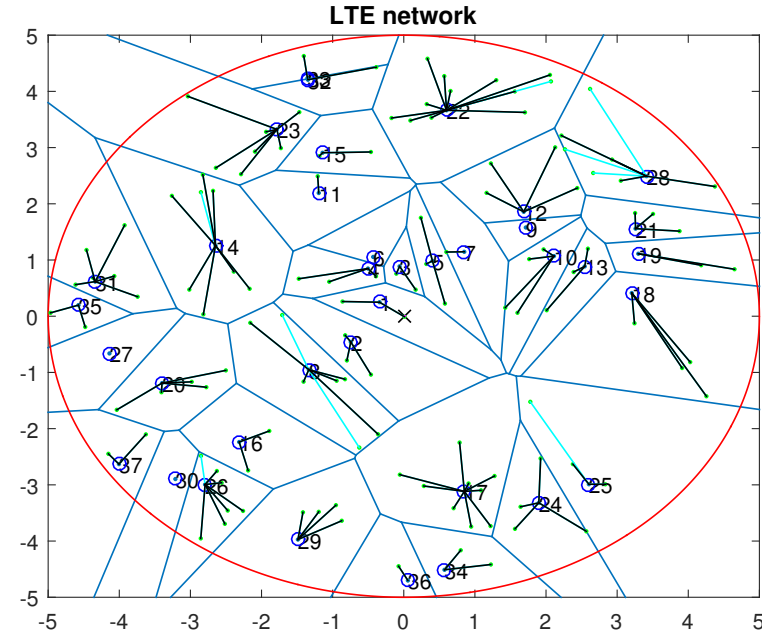

Fig. 1: Modeled broadband network with a PPP describing the position of BSs, users and cells

where $P_{t}$ is the transmitted power, $h_{m, i}$ is a random variable representing the channel for user $m$ and RB $i$, with exponential distribution of mean $1 / \mu$ i.e. $h \sim \exp (\mu)$. $\alpha$ represents the path loss exponent, $f$ is the operating frequency in $\mathrm{Hz}, \mathrm{c}$ is the speed of light and $r_{m}$ is the distance between the user $m$ and its serving BS. the noise power at the receiver is considered to be a constant and is denoted by $\sigma^{2}$, while the interference power at user $m$ for RB $i$ expressed as:

$$
I_{m, i}=\sum_{j \in \Phi / u_{m}} P_{t} h_{m, i, j}\left(\frac{4 \pi f}{c}\right)^{-\alpha} d_{m, j}^{-\alpha}
$$

where $\Phi$ is the set of all BS transmitters, $u_{m}$ denotes a user $m, d_{m, j}$ is the distance between the receiver $m$ and the interfering transmitter $j$ and $h_{m, i, j}$ denotes a random variable that represents the channel between user $m$ and the $j t h$ transmitter, for RB $i$. Eq. 1 could then be reduced to:

$$
\gamma_{m, i}=\frac{h_{m, i} r^{-\alpha}}{\tilde{\sigma}^{2}+\sum_{j \in \Phi / b_{0}} h_{m, i, j} d_{m, j}^{-\alpha}}
$$

We introduce a normalized noise variance $\tilde{\sigma}$ according to path loss given by:

$$
\tilde{\sigma}^{2}=\frac{\sigma^{2}}{P_{t}\left(\frac{4 \pi f}{c}\right)^{-\alpha}}
$$

The capacity of a single RB $i$ for a user $m$ will then be as following:

$$
C_{m, i}^{R B}=B_{R B} \log _{2}\left(1+\gamma_{m, i}\right)
$$

where $B_{R B}$ is the bandwidth of a single $\mathrm{RB}$, usually equal to $180 \mathrm{KHz}$ in LTE networks

In this work, we assume that the capacity of user $m$ to be the sum of capacities of the RBs assigned to this user. It is given by:

$$
C_{m}^{\text {user }}=\sum_{i=1}^{R B_{\max }} C_{m, i}^{R B} a_{i, m}
$$


where $a_{i, m}$ is a binary variable that is equal to 1 if $\mathrm{RB} i$ is assigned to user $m$, and equal to 0 otherwise.

Similarly, the capacity of a cell is defined as the total capacities of all users attached to this cell and will be defined as follows:

$$
C_{n}^{\text {cell }}=\sum_{m \in \mathscr{M}_{n}^{\text {cell }}} C_{m}^{\text {user }} b_{m}
$$

where $\mathscr{M}_{n}^{\text {cell }}$ represents the set of all users in a cell $n$, and $b_{m}$ represents a binary variable indicating whether user $m$ is properly receiving the linear service or not, i.e. whether the transmitter succeeds or fails to assign enough RBs so that $C_{m}^{u s e r}$ exceeds $C^{r e q}$.

\section{Performance metrics}

In order to evaluate the different approaches of a linear service, we introduce the average service success rate. The service SR $\eta^{s}$ is defined as the ratio between the number of users that have the access to the service to the total number of users in the service area. In the case of overload, the network will deny the service access. In a cell, the success rate can then be stated as:

$$
\eta_{n}^{\text {cell }}=\frac{\text { Number of served users }}{\text { Number of users in the cell }}
$$

which can be also expressed as

$$
\eta_{n}^{\text {cell }}=\frac{\sum_{m \in \mathscr{M}_{n}^{\text {cell }}} b_{m}}{M_{n}^{\text {cell }}}
$$

where $M_{n}^{\text {cell }}$ is the number of users in cell $n$ Hence, the success rate of the system is given by:

$$
\eta^{s}=\frac{\sum_{n=1}^{N} \sum_{m \in \mathscr{M}_{n}^{\text {cell }}} b_{m}}{M}
$$

It should be noted that other metrics are often used to evaluate a resource allocation scheme, like system capacity and fairness index, or Jain's fairness index [8] in precise. However, system capacity has no significant importance in linear services compared to SR. In addition, Jain's fairness index is highly correlated with the served (non-zero) users, i.e. SR, and its information would be redundant.

\section{PROBLEM FORMULATION AND PROPOSED ALGORITHM}

\section{A. Problem formulation}

The aim of this work is to maximize the success rate defined in Eq. (9), with a constraint on the minimum allowed capacity for a served user, and another constraint on a maximum number of users allocated with the same RB, limiting it to only one user at a single time slot. The optimum success rate of the system is achieved when all the cells are also at the optimum success rate, so we define following optimization problem for each cell:

$$
\begin{array}{ll}
\max _{\mathbf{a}} & {\left[\sum_{n=1}^{N_{B S}} \sum_{m \in \mathscr{M}_{n}^{\text {cell }}} b_{m}\right]} \\
\text { subject to } & \sum_{i} a_{i, m} \leqslant R B_{\max } \\
& C_{m}^{\text {user }}=\sum_{i=1}^{R B_{\max }} C_{m, i}^{R B} a_{i, m} \\
\forall n & \sum_{m \in \mathscr{M}_{n}^{\text {cell }}} a_{i, m} \leqslant 1 . \\
& b_{m}= \begin{cases}1, & \sum_{i=1}^{R B_{m} a x} a_{i, m} C_{i}^{R B}, m \geq C^{r e q} \\
0, & \text { Otherwise. }\end{cases}
\end{array}
$$

where a denotes the matrix holding all $a_{i, m}$. Recall that $b_{m}$ represents a binary variable that is equal to 1 if user $m$ is connected to its corresponding BS, and equal to zero otherwise. Also recall that $a_{i, m}$ represents a binary variable that is equal to 1 if the RB $i$ is allocated to user $m$ in its corresponding cell, $C_{m}^{u s e r}$ represents the achieved capacity of user $m, C^{r e q}$ is the minimum required capacity for a user to be connected, $R B_{\text {max }}$ represents the maximum number of resource blocks available in a cell, and $\mathscr{M}_{n}^{\text {cell }}$ is the set of all users in cell $n$.

In the problem described above, $b_{m}$ depends on several other variables in the system, so it holds information on SINR, and consequently on the distance from base station $r$, the channel and the interference. It also depends on the set of allocated $\mathrm{RBs}$ to the corresponding user, since each RB has different SINR even for the same user and same base station. The limited number of RBs in a cell increases the complexity of the problem. Indeed, whenever a RB is assigned to a user, it won't be available for allocation to other users, hence to the RB pool for the next users, and consequently, options and diversity are reduced. This correlation between users capacities and variable dependencies indicates that the complexity of the above problem makes its analytical solution, without losing generality, prohibitive. We should mention that this algorithm is by nature integer binary problem, hence MIP solutions could be proposed. However, again due to its nature, it is very difficult to propose an adequate analytical solution. Rather, the choice of $a_{i, m}$ and consequently $b_{m}$ could be based on an algorithm that leads to maximizing the success rate. Our proposed algorithm is described next.

\section{B. Proposed algorithm description}

The linear nature of the services is the basic motivation in our work. In non-linear services, QoS for a user increases with its link capacity. Hence, most of the work in this domain has dealt with the maximization of the system's overall capacity as a goal. Alternatively, when linear service is delivered, a certain level capacity for a user is enough for a proper reception. Hence, the main idea of our proposed algorithm is to fix a minimum threshold for the user capacity and try to maximize the success rate and increase the index of fairness, while avoiding the usage of unnecessary or not beneficial RBs. 
Due to the limited number of RBs, maximizing the number of connected users could be achieved by allocating the exact number of RBs for a user just to reach the required capacity $C^{r e q}$. The proposed algorithm relies on assigning the RBs to the users having the best conditions hence, increasing the expected number of served users. In fact, assigning the RBs to users with worst condition first will drain the number of RBs offered to other users, since such users could be resource hungry. In addition, the selection of the RBs with best channel coefficients will reduce the required number to reach $C^{r e q}$, and consequently enhance the number of connected users by increasing available RBs.

The proposed LSORAS algorithm for each cell is described in Alg. 1.

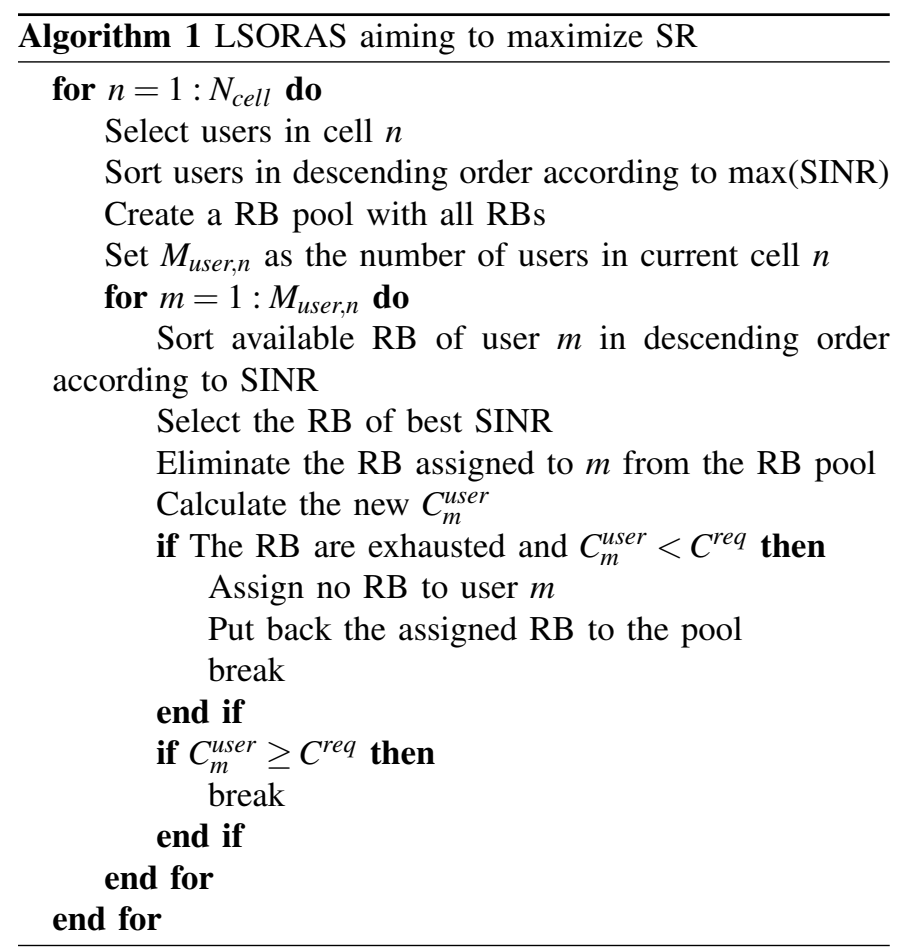

Such algorithm satisfies all the points mentioned earlier and consequently leads to the best use of the RBs available in a given distribution. In addition, this algorithm will not assign useless RBs for a user in two cases: the case when the capacity achieved by a user exceeds $C^{r e q}$, and the case when assigning the available number of RBs will not satisfy the given user constraint. This property will save RBs to other services within the same cell, i.e. implicitly turn off the unused RBs for power saving.

\section{Optimal Solution By Branch and Cut}

In order to assess the performance of LSORAS algorithm, a comparison with the optimal solution was achieved. Since our problem is an mixed integer (binary) programming problem, branch and cut algorithm was also implemented. Branch and cut [9] is a combinatorial optimization method that solves MIP problems $_{i}$ It is based on two main techniques:
1) Branch and bound [10]: a tree traverses all possible solutions for each variable while calculating lower and upper bound of the possible solutions. The latter are used to eliminate some branches of the tree which cannot produce an optimal solution, hence reducing complexity[11].

2) Cutting planes [12]: used to narrow the linear Programming relaxation. Once a solution is found, it is checked if its an integer. If it is not the case, it is guaranteed to have a separation inequality that separates the optimum from the convex hull.

The MIP problem is solved using a regular simplex algorithm while relaxing the integer constrains, which will result in obtaining a non-integer solution as a first step. The second step is to find a additional linear constraints (by cutting plane algorithm) that is violated by the non-linear solution found in first step, but are satisfied by all integer points. Adding those constrains will lead to a different less fractional solution. the same procedure is repeated until an integer solution is found, or until no more cutting constraints could be found. In this case, branching is done for possible integer solution for an element of the vector. after branching, smaller problems are created, and the same procedure is repeated, until the complete integer vector is found. The $\mathrm{BaC}$ algorithm is out of order in the paper and left to the reader for more information. Reader can refer to $[13,14]$.

However, $\mathrm{BaC}$ is guaranteed to reach an optimal solution if it is implemented correctly and given enough time. For this reason, it will be used as a benchmark for LSORAS in terms of performance.

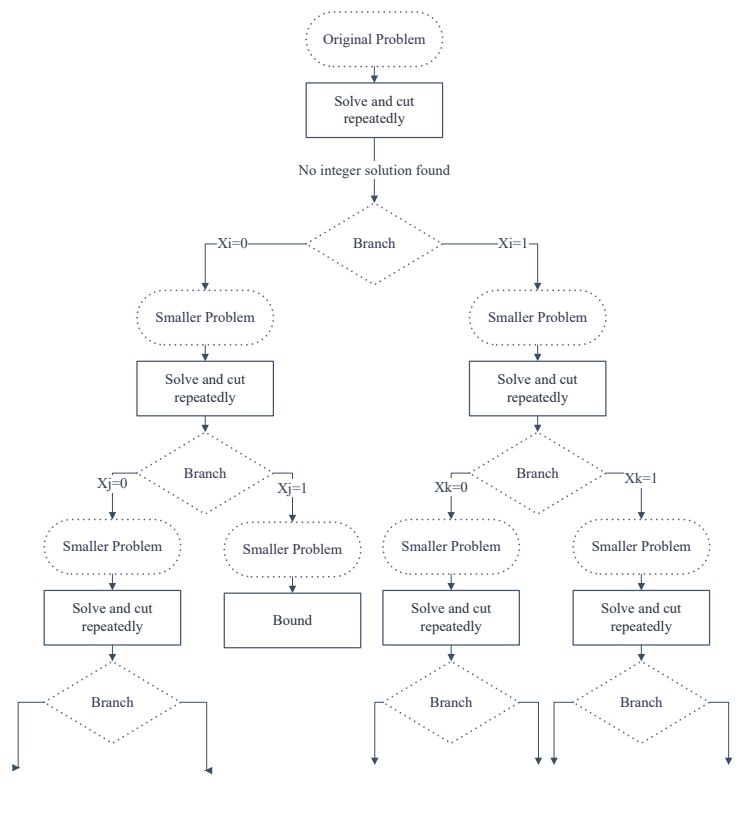

Fig. 2: Example $\mathrm{BaC}$ procedure 


\section{Simulation Results}

In this section, numerical simulation of the proposed algorithm is presented. The results are compared to MT, RR and $\mathrm{PF}$ algorithms, and most importantly to the optimal results provided by branch and cut algorithm. In addition, the effect of the user density and required capacity on the performance of the proposed algorithm is studied.

\section{A. Simulation setting}

For the simulation, the broadband model was created with suitable PPP density to have around $25 \mathrm{BS}$ in the service area, i.e. $\lambda=1 / \pi$. For cell boundaries, a Voronoi tessellation is used. The user distribution was also set according to a PPP with $\lambda=5 / \pi$ leading to almost 125 users. The target capacity or $C^{r e q}$ is $1.5 \mathrm{Mbps}$. Table I gives the main simulation parameters used.

TABLE I: Simulation parameters

\begin{tabular}{|l|l|}
\hline \multicolumn{2}{|c|}{ simulation parameters } \\
\hline$\lambda_{B S}$ & $1 / \pi$ \\
Isotropic $P_{t}$ & 1200 Watt \\
$R B_{\max }$ (available RB) & 100 \\
LTE BW & $20 \mathrm{MHz}$ \\
RB BW $B_{R B}$ & $180 \mathrm{kHz}$ \\
Path loss exponent $\alpha$ & 3 \\
Operating frequency $f$ & $2110 \mathrm{MHz}$ \\
Radius of service Area & $5 \mathrm{~km}$ \\
\hline
\end{tabular}

In order to implement the $\mathrm{BaC}$ optimal solution, $\mathrm{AIMMS}^{\circledR}$ [15] software has been used. AIMMS is designed for modeling and solving large-scale optimization and scheduling problems, particularly MIP using BaC.

\section{B. Probability of coverage}

Figure 3 shows a comparison between the proposed LSORAS algorithm with MT, PF, RR, and with the optimal solution produced by $\mathrm{BaC}$ algorithm, in terms of Complementary Cumulative Distribution Function (CCDF). The CCDF is seen as the probability of coverage, i.e. the probability of a user to have a capacity that exceeds a certain value. Obviously, LSORAS outperforms MT, PF, and RR. For a user capacity threshold at $1.5 \mathrm{Mbps}, 89 \%$ of the users succeed to receive the service properly, compared to around $64 \%$ with RR and $\mathrm{PF}$, and around $31 \%$ for Max Throughput. LSORAS curve has a flat shape below 1.5 Mbps due to the fact that, as per the proposed allocation, no user will have a capacity between zero and $C^{r e q}$; it is either zero, $C^{r e q}$, or some value slightly higher. Results also show that LSORAS is only $1.8 \%$ below the optimal solution, which is not significant compared to the reduction in computation, and the number of the resource blocks used.

Compared to the computationally expensive branch and cut, especially at a high number of users, LSORAS is a much less expensive method that achieves comparable results. From another perspective, LSORAS uses only a part of the RBs available, compared to a complete consumption of resources in MT, RR, PF solutions. For $C^{r e q}=1.5 \mathrm{Mbps}$, and 5:1 users to $\mathrm{BS}$ ratio, only $47 \%$ of the available $100 \mathrm{RBs}$ were used, compared to $100 \%$ in MT, PF, RR and branch and cut. This means that the system saves around $53 \%$ of the resources, which could be assigned to other services or turned off to reduce power consumption.

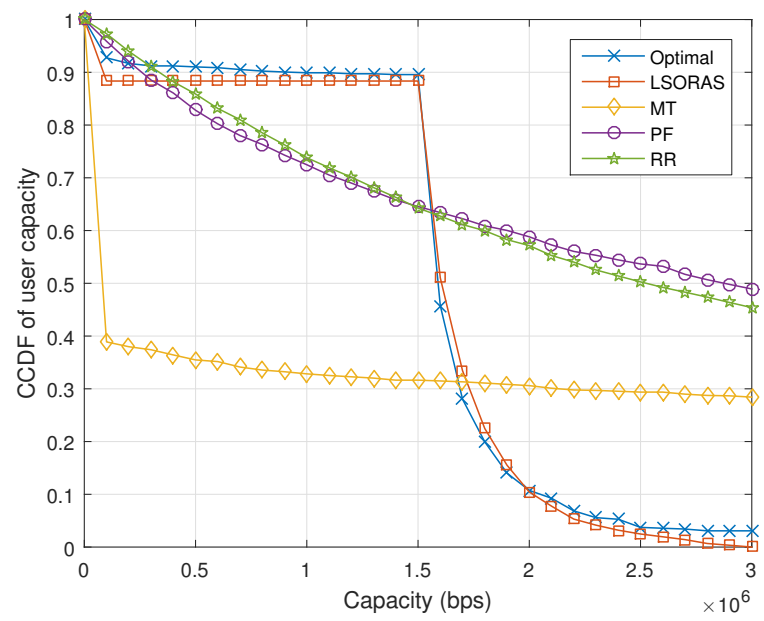

Fig. 3: CCDF of user capacity for 5:1 user to base station ratio, $C^{r e q}=1.5 \mathrm{Mbps}$

\section{Effect of user density}

Figure 4 shows the variation of success rate with respect to the number of users in a service area of around 100 base stations. The results show that the success rate with MT, PF and RR methods dramatically decays with the increase of the number of users. On the other hand, LSORAS maintains higher success rates even with a load ratio 30:1 users per BS, while the decay is not as severe as with the other methods. The optimal solution provided by branch and cut keeps a small advantage over LSORAS. However, for a large number of users, the $\mathrm{BaC}$ computation becomes very expensive in terms of memory, power and time compared to LSORAS.

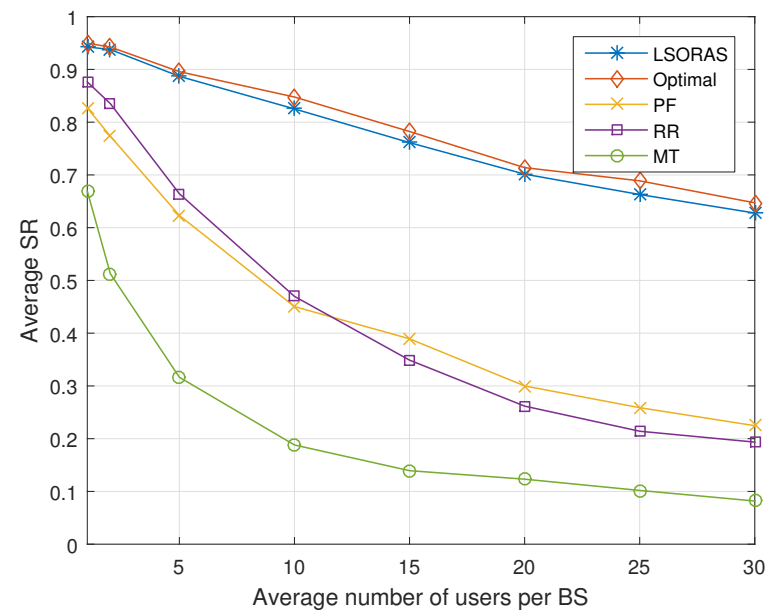

Fig. 4: Average SR vs the number of users for $100 \mathrm{BS}$ in the service area, with $C^{r e q}=1.5 \mathrm{Mbps}$ 


\section{Effect of user required capacity}

Figure 5 shows the variation of success rate as a function of required capacity. The results show that LSORAS proves superiority over MT, PF, and RR, even when the required user capacity is as high as $5 \mathrm{Mbps}$. Results show a smooth slow decay in SR, almost linearly from values slightly lower than $100 \%$ at $0.5 \mathrm{Mbps}$, to around $65 \%$ at $5 \mathrm{Mbps}$, compared to steeper decay in PF and RR. Those results show the robustness of LSORAS against higher QoS.

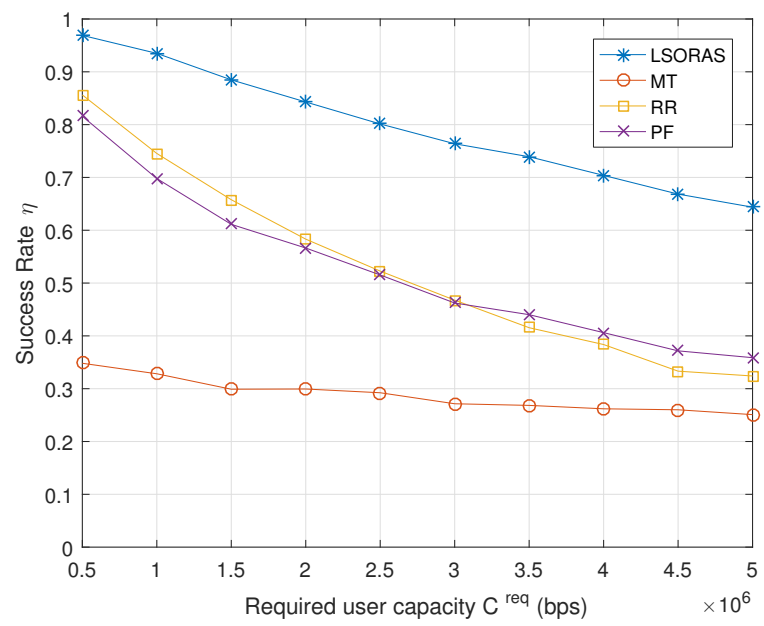

Fig. 5: Average SR vs the required capacity $C^{r e q}, 5: 1$ users to $\mathrm{BS}$ ratio

\section{CONCLUSiON}

This work has considered the case of linear services provided by a broadband network. We designed a new linearservice oriented resource allocation strategy (LSORAS) which manages to maximize the number of served users in a certain service area. Numerical results have been presented to verify the effectiveness of the proposed algorithm. Comparison to the optimal solution in terms of success rate showed that LSORAS results are very close to optimal, with significantly less computational resources. The results have shown a significant increase in success rate compared to other algorithms. The results also showed the robustness of the algorithm against user density and higher required QoS. It also showed that unlike the other algorithms, LSORAS manages to save a significant portion of RBs for other services, or power reduction. Undoubtedly, this work could be easily extended to broadcast and multicast mode and will be considered in future research directions.

\section{ACKNOWLEDGEMENT}

This work has received a French state support granted to the Convergence TV project through the $20^{\text {rd }}$ FUI (transverse inter-ministry funding) program. The authors would also like to thank the "Image \& Réseaux" and "Cap Digital" French business clusters for their support of this work.

\section{REFERENCES}

[1] T.Jr. Barnett et al. Cisco Visual Networking Index (VNI) update global mobile data traffic forecast. 2015. URL: http://graphics8.nytimes.com/packages/pdf/technology/ cisco-mobile-forecast.pdf.

[2] G. Piro et al. "Two-level downlink scheduling for realtime multimedia services in LTE networks". In: IEEE Transactions on Multimedia 13.5 (2011), pp. 10521065.

[3] H. Zhang et al. "Resource allocation in spectrumsharing OFDMA femtocells with heterogeneous services". In: IEEE Transactions on Communications 62.7 (2014), pp. 2366-2377.

[4] A.S. Mohamed, M Abd-Elnaby, and S.A. El-Dolil. "Self-organised dynamic resource allocation scheme using enhanced fractional frequency reuse in long term evolution-advanced relay-based networks". In: IET Communications 10.10 (2016), pp. 1163-1174.

[5] N. Kumar, A. Ahmad, and D. Prasad. "Survey of downlink control channel resource allocation techniques in LTE”. In: 2015 Annual IEEE India Conference (INDICON). 2015, pp. 1-5.

[6] Z. Tang. Traffic scheduling for LTE advanced. 2010.

[7] D. Gesbert. "A Channel Predictive proportional fair schedulaing algorithm". In: IEEE 6thWorkshop, SPAWC (2005), pp. 620-624.

[8] R. Jain, D.M. Chiu, and W.R. Hawe. "A quantitative measure of fairness and discrimination for resource allocation in shared computer system". In: ACM Transaction on Computer Systems cs.NI/9809.DEC-TR-301 (1984), pp. 1-38.

[9] M. Padberg and G. Rinaldi. "A Branch-and-Cut algorithm for the resolution of large-scale symmetric traveling salesman problems". In: SIAM Review 33.1 (1991), pp. 60-100.

[10] A. H. Land and A. G. Doig. "An automatic method of solving discrete programming problems". In: Econometrica 28.3 (1960), pp. 497-520.

[11] J.Clausen. Branch and Bound algorithms-principles and examples. Tech. rep. University of Copenhagen, 1999.

[12] H. Marchand et al. "Cutting planes in integer and mixed integer programming". In: Discrete Applied Mathematics 123.1 (2002), pp. 397 -446.

[13] J.E.Mitchell. "Branch-and-cut algorithms for combinatorial optimization problems". In: Handbook of applied optimization (2002), pp. 65-77.

[14] T.Achterberg, T.Koch, and A.Martin. "Branching rules revisited". In: Operations Research Letters 33.1 (2005), pp. $42-54$.

[15] AIMMS B.V., Haarlem, The Netherlands. AIMMS. Version 4.37. 2017. URL: http://www.aimms.com/. 